\title{
Enabling Cooperation between ISPs and P2P Systems toward IPTV Service Delivery
}

\author{
Mubashar Mushtaq ${ }^{1,2}$ and Toufik Ahmed ${ }^{1}$ \\ ${ }^{1}$ CNRS LaBRI Lab. - University of Bordeaux, 351, Cours de la Libération, Talence, 33405, France \\ ${ }^{2}$ Dept. of Computer Science, Quaid-i-Azam University, Islamabad, 45320, Pakistan \\ Email: \{mushtaq, tad\}@labri.fr
}

\begin{abstract}
The wide adoption of the P2P networks for the deployment of IPTV services at large scale seems very fascinating. However, it has started exposing the other side of canvas that is not acceptable for the provisioning of QoS for P2P applications. It is even more frustrating for the ISPs due to inefficient utilization of network resources. In this paper, we investigate the conflicting objectives of P2P applications \& ISPs and we propose a framework enabling better interaction among entities involved in the IPTV service delivery mechanism. This interaction is facilitated by a topology-aware overlay network organization driven by the ISPs that is used to organize the participating peers within the network. The proposed ISPs-driven overlay organization facilitates the multi-attribute based sender peers selection mechanism and thus ensures the efficient utilization of available resources in ISPs domain and guarantees better QoS for the P2P applications. The framework is evaluated using the networks simulations to conform its viability. The obtained results show a noticeable improvement in the received QoS for the P2P applications and enable the efficient utilization of the available network resources to benefit the ISPs.
\end{abstract}

\section{INTRODUCTION}

During the last decade, the peer-to-peer (P2P) networking has gained tremendous attentions and is widely adopted for the deployment of many distinct IPTV and other video services delivery to a large scale. The tremendous success of this infrastructure is due to the exceptional characteristics of P2P systems that include: scalability, dynamicity, heterogeneity, collaboration, and anonymity. However, the recent developments in this field have forced the research communities to investigate the other side that is not very encouraging. Generally, the IPTV service delivery in P2P network is provisioned by the $\mathrm{P} 2 \mathrm{P}$ applications themselves to estimate end-to-end ( $2 \mathrm{e}$ ) network's conditions which are agnostic to network topology. The objective of P2P applications is to receive the video contents more quickly and with high Quality of Service (QoS). On one hand, this network oblivious scenario let the contents pass through the network links that may be costly or may unable to support large amount of content. This results in the degradation of QoS at receiver-end. On the other hand, this situation is frustrating for the ISPs, who are missing the opportunities to earn revenues from the external traffic passing through their physical networks. The situation becomes more provoking when ISPs have to deploy more resources to offer their own services. This situation results into continuous battles between these entities where ISPs intend to restrict P2P traffic passing through their networks and $\mathrm{P} 2 \mathrm{P}$ application providers try to evade from being captured. Thus, we need to bring these entities towards a common point to cease this no-winner war.

In $\mathrm{P} 2 \mathrm{P}$ applications, participating peers are organized in virtual overlay networks. These overlay networks can be categorized to: "network-oblivious overlays" where links among neighbors are based on identifiers in logical space, "proximity-aware overlays" that incorporate physical closeness of nodes and "network-aware overlays" that exploit locality for the underlying routing strategy [1].

In this paper, we extend the "network-aware overlays" concept by enabling better interaction among the ISPs and the P2P applications for the graceful utilization of available resources and respecting the objectives of entities involved in the IPTV service delivery mechanism. We propose a framework that is based on ISP-driven overlay organization because ISPs have the complete information of the underlying physical networks that is helpful to build overlay network more efficiently. The ISP-driven overlay networks are further used for locating the best sender peers on the basis of multi attributes for efficient IPTV service delivery. The ultimate objective of this framework is to optimize the application level traffic with efficient collaboration between ISPs and P2P applications to offer network-aware video services.

The rest of the paper is organized as follows: section II presents the proposed collaborative framework. Section III presents multi-attribute based peer selection mechanism for IPTV service delivery. Section IV presents the performance evaluation and section $\mathrm{V}$ presents a brief overview of related works. Finally section VI concludes the paper and highlights some of the future perspectives of the study.

\section{COOPERATIVE FRAMEWORK FOR ISPS AND P2P APPLICATIONS}

This section presents the problem description along with the proposed framework for better cooperation between ISPs and $\mathrm{P} 2 \mathrm{P}$ applications that is further used to organize different peers in overlay network for IPTV video service delivery.

\section{A. Problem Description}

The aim of this cooperative framework is to ensure the objectives of all entities involved in the IPTV video service delivery mechanism. In this mechanism, the ISPs plays an important role by providing the information related to the underlying physical network that is further used to organize the participating peers into overlay networks. A receiver peer 
selects different sender peers from this ISP-driven network in order to receive requested video contents with high QoS.

Generally, ISPs have two major roles in current Internet architecture including: physical network providers and service providers. In this paper, we are more concerned with over the top IPTV video services delivery that is not supported by ISPs themselves. These services are provided by external service providers like $\mathrm{P} 2 \mathrm{P}$ applications that use the underlying physical network of ISPs even without any permission of certain ISPs. ISPs tries to restrict such traffic passing through their networks, however it is not trivial because many P2P applications use different protocols and encrypted ports to evade from being captured. In the current P2P service model, the organization of participating peers into overlay networks is mostly receiver-centric, where receiver peer gathers the information of other peers and organizes them in overlay networks on the basis of self agnostic and reverse engineering e2e measurements.

\section{B. Network Model}

Let $G_{u}=\left(P_{u}, V_{u}\right)$ ("u" for underlay) represents the underlying physical network and $G_{o}=\left(P_{o}, V_{o}\right)$ ("o" for overlay) represents the overlay network that is used to organized participating peers in virtual overlay networks. $G_{o} \subseteq G_{u}$ because overlay networks are composed of sub-set of peers that are willing to participating in any specific service delivery mechanism. In this network, $p_{i} \in P_{i}$ denotes the set of participating peers whereas $\mathrm{v}_{\mathrm{i}} \in \mathrm{V}_{\mathrm{i}}$ denotes the links between these peers forming overlay network.

The proposed overlay organization is driven by the ISPs that have clear knowledge of underlying network topology, geographical positions of the peers, and the information of available networks resources (access bandwidth), link capacity at access networks" $\operatorname{cap}_{a}$ ", and "e2e" link capacity" $\operatorname{cap}_{e 2 e}$. Let "loc $\left(P_{i}\right)$ " and "cap $\left(P_{i}\right)$ " represents the geographical location of the peers and the available link capacity of access network between peers respectively. This set of information for corresponding candidate peers is propagated to the $\mathrm{P} 2 \mathrm{P}$ application for the overlay organization. The link capacity in access networks of intra-domains influence the IPTV service delivery. We use trackers as special entities for the interaction between entities. A brief interaction among these entities is shown in Fig. 1. The objective of locality-aware organization is to minimize the " $e 2 e$ " delay for the video contents delivery that plays vital role in improving the overall QoS.

\section{Contrasting Objective Functions}

ISPs and $\mathrm{P} 2 \mathrm{P}$ applications providers have contrasting objectives that need to be addressed to protect the interests of all the entities involved in video service delivery. These contrasting objectives are presented as under:
- ISPs Objectives: We denote ISPs objective function by $\mathrm{f}_{1}$ that intends to maximize the utilization of available network resources to offer more services over available network resources.

- P2P Applications Objectives: P2P application providers intend to improve the QoS by maximizing the video throughput and minimizing the time required to receive the full video contents and the objective function is denoted by $\mathrm{f}_{2}$.

- ISPs \& P2P Cooperative Objective: The overall objective of this study is to propose a mechanism that enables better cooperation between ISPs and P2P application providers to maximize the objectives of both entities, i.e. maximizing $\mathrm{f}_{1} \& \mathrm{f}_{2}$.

\section{ISP-driven Framework to Enable Cooperation}

The proposed framework targets the over the top video "OTT" (content coming from outside of the operators' network) IPTV contents delivery. P2P application has little knowledge of the underlying physical network topology, and it chooses the content delivery paths based on self estimated measurements and statistic that may cause traffic scattering. Hence, the actual media content unnecessarily traverse multiple links within network to reach the targeted clients. This delivery process imposes higher load on network backbone and such traffic pattern may cause imbalance between peers, leading to potential violation of peering agreements between network providers.

The network-aware ISP-driven overlay network constitute of candidate peers willing to participate in specific video service delivery. There are two types of candidate peers (i) permanent nodes, and (ii) fleeting nodes. The permanent nodes are usually hosted by ISPs/service providers themselves and may include: "superpeer" and "caching nodes". These nodes remain available in the network for the service delivery. The fleeting nodes include "seeders", "leechers", and “mercenaries”. These nodes are highly volatile and may join the P2P networks for seeking any requested service and may leave the network without any prior notification. However, "mercenaries" are paid by the service providers for being available in the network due to their capabilities to serve the subsequent video service requests.

A receiver peer intending to receive a video service may select a number of sender peers from the overlay network on the basis of its subscription. For example, "free riders" or "ordinary" consumers may connect to "seeders" or "leechers", whereas, the "valued" customers are guided to choose more “mercenaries” and permanent nodes enabling better QoS for the offered video service. The service consumer's status is regularly updated using different economical models like "titfor-tat” on their usage and contribution behavior.

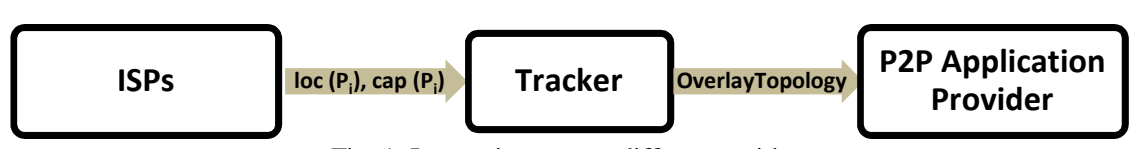

Fig. 1. Interaction among different entities. 
Figure 2 presents a comprehensive view of the proposed framework that is composed of different entities including management portal, overlay plane, and control plane that have potential role in this application level collaborative traffic optimization between ISPs \& P2Ps.

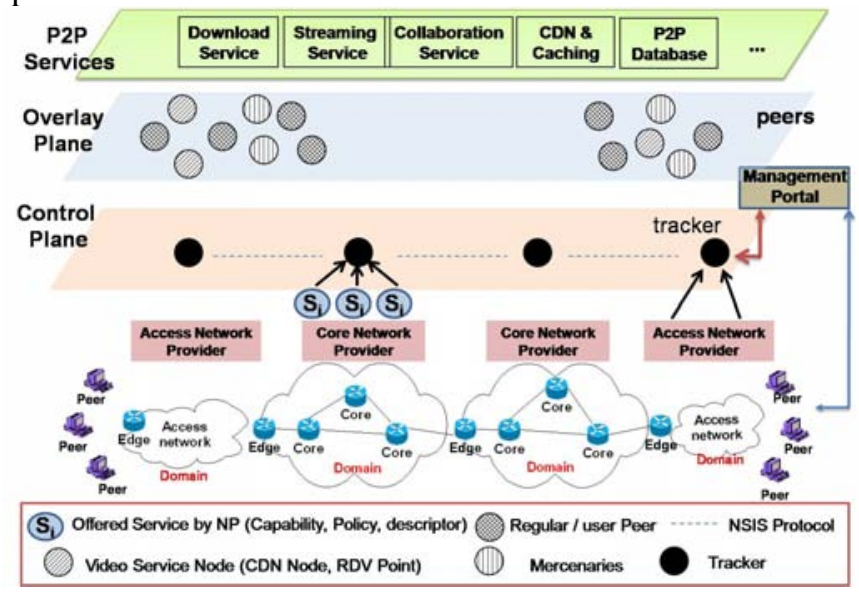

Fig. 2. ISPs \& P2P Cooperative Framework for Video Services Delivery.

- Management Portal: The management portal provides an entry/rendezvous point for the new peer joining the network that is accessible through a webpage. The management portal returns a list of active trackers present in the network that allow interaction among the P2P application providers and network providers.

- Overlay Plane / ISP-driven Overlay: This ISP-driven overlay plane provides a topology-aware overlay organization of participating peers. These peers have distinct roles including: ordinary peers joining P2P network for different services, video service nodes (CDN nodes, RDV points), and mercenaries. Video service node is special peer that act as the rendezvous point for the new peers joining the overlay and can also be used for the caching of the most popular video content. This overlay plane is further used for the deployment of different P2P services.

- Control Plane: Control plane is another important entity of the proposed framework that is composed of the different trackers enabling the cooperation between underlying network domains and the P2P applications. These trackers communicate with the network provider domains and this information is further used for the peer's organization in the overlay networks. Each network provider domain has an independent tracker that is responsible to provide underlying network topology information for the overlay organization. Beside from this vertical interaction, these trackers have horizontal interaction to share the inter-ISPs information.

We have described earlier that peers forming the overlay network are belonging to different substrate networks within same ISP domain, thus information among these substrate networks is shared by a designated entity called "Tracker". The trackers enable the horizontal (inter-substrate networks) and vertical (between physical networks and P2P application providers) communications using the NSIS protocols [2]. These trackers have distinguished role in this cooperative framework for locating the best peer for video service delivery that is described in following sub-section

\section{IPTV SERVICE DELIVERY OVER COOPERATIVE FRAMEWORK}

The IPTV service delivery over cooperative framework is influenced by the multi-attribute based sender peer selection and utilizing the H.264-Scalable Video Coding (SVC) that are described in the following sub-sections.

\section{A. Best Peers Selection Based on Multi Parameters}

The selection of best sender peers is an active research problem in content delivery over P2P networks. There have been different solutions presenting different parameters for organizing the peer into overlay network and thus, locating the best peers. These mechanisms include receiver-centric peer selection, server-centric selection, locality-aware selection, semantic-aware selection, and different incentive mechanisms etc. In general, peer selection is based on the basis of a single parameter. However, we need to identify a set of multiparameters for the overlay organization following the best peer selection mechanism, because no single parameter is sufficient to enable service delivery over large-scale networks [3]. In the proposed framework, ISPs provide the information to build the overlay topology and the corresponding information includes the information of available peers, offered video quality by these peers, information related to aggregation nodes, available bandwidth over " $e 2 e$ " network path, and the geographical information of the peers. This ISPdriven overlay topology is further used by any receiver peer to select a set of sender peers in order to receive the requested video contents/services. Bad selection in sender peers not only results into the degradation of the QoS but also wastes the network resources in ISPs domains. In this paper, we focus to use TOPSIS (Techniques for Order Preference by Similarity to an Ideal Solution) [4] method which is a multiple criteria method to identify solution from finite set of points where the chosen points should have the "shortest" distance from the positive ideal solution and the "farthest" distance from the negative ideal solution.

In the framework, trackers are responsible to apply this technique to determine positive ideal and negative ideal alternative values that are further used for the best peer selection. The " $e 2 e$ " available bandwidth capacity among sender and receiver peers, " $e 2 e$ " packets arrival delay, total number of Hops, "e2e" round-trip-time (RTT), and geographical distance may be the possible criteria for applying TOPSIS technique. RTT between receiver and sender peers is consider good parameter for latency estimation that can also be used for the geographical distance measurements. Generally, for the real time services like video streaming higher bandwidth, less number of hops, lower RTT, lower packets drop ratio, etc. are considered ideal options. 
Table 1. Multi Attributes for Best Peers Selection for Cooperative IPTV Service Delivery.

\begin{tabular}{l|ccccc}
\hline & Bandwidth & Latency & RTT & Geo. Distance & \# of Hops \\
\hline Option 1 $\left(f_{1}^{+}, f_{2}^{+}\right)$ & + & - & - & - & - \\
Option 2 $\left(f_{1}^{+}, f_{2}^{-}\right)$ & $+/-$ & $+/-$ & $+/-$ & $+/-$ & $+/-$ \\
Option 3 $\left(f_{1}^{-}, f_{2}^{+}\right)$ & $+/-$ & $+/-$ & $+/-$ & $+/-$ & $+/-$ \\
Option 4 $\left(f_{1}^{-}, f_{2}^{-}\right)$ & - & + & + & + & + \\
\hline
\end{tabular}

Table 1 presents different attributes and the possible options that tracker may takes into consideration for the ranking of available peers while organizing them into overlay plane. Here, we have given a limited list of potential attributes that may be considered, however there might be many other attributes like BGP routing information, geographical distances, " $A S$ " domain information. In this table, we have presented four different options to find the appropriate organization of peers into overlay plane while maximizing the objective functions of ISPs $\left(f_{1}\right)$ and objective function of P2P applications $\left(f_{2}\right)$ as shown. In Table 1 "Option 1" presents the best values where both $\left(f_{1}\right.$ and $\left.f_{2}\right)$ are maximized and "Option 4" gives the worst results for both functions.

These set of values given in the matrix are further classified into positive " $j^{+"}$ (more is better) and negative $" j^{-"}$ attributes (less is better) while applying the multi criteria TOPSIS model. $j^{+} \in$ bandwidth, throughput,network_resources, $\ldots$ and $j^{-} \in$ latency,jitter, geo. distance, RTT, \#of Hops .... .

Let $A^{+}=\left\{x_{1}^{+}, x_{2}^{+}, \ldots, x_{n}^{+}\right\}$where $x_{i}^{+}=\left\{\max _{i}\left(x_{i j}\right)\right.$ if $j \in$ $j^{+} ; \min \left(x_{i j}\right)$ if $\left.j \in j^{-}\right\} \quad \& \quad A^{-}=\left\{x_{1}^{-}, x_{2}^{-}, \ldots, x_{n}^{-}\right\}$where $x_{i}^{-}=\left\{\min _{i}\left(x_{i j}\right)\right.$ if $j \in j^{+} ; \max _{i}\left(x_{i j}\right)$ if $\left.j \in j^{-}\right\}$represents the positive and negative solutions for the utility function with respect to the possible set of attributes and are achieved by assigning different weights for each attribute in different options. The weights depend upon the offered service and may be negotiated between ISPs and P2P applications. For example, video-on-demand (VoD) applications require high bandwidth and live streaming applications have low latency requirements. These sets of attributes are further used for calculating the separation from the positive and negative ideal solutions, such that: $\forall i \in 1,2, \ldots, m$,

$X^{+}=\left[\sum\left(x_{i}^{+}-x_{i j}\right)^{2}\right]^{1 / 2} \quad, \quad X^{-}=\left[\sum\left(x_{i}^{-}-x_{i j}\right)^{2}\right]^{1 / 2} \quad$ and finally the relative closeness $\left(C_{i}^{+}\right)$to the ideal solution is determined: $C_{i}^{+}=\frac{X_{i}^{-}}{\left(X_{i}^{+}+X_{i}^{-}\right)} \approx 1$, where $0<C_{i}^{+}<1$ and the values closet to " 1 " are selected for the ideal solutions. Tracker facilitates any receiver peer for selecting the best sender peer by providing these estimates.

\section{B. IPTV Service Delivery}

In the proposed framework, the overlay organization of participating peers is ISPs-driven, however the actual IPTV video content delivery is receiver-centric in which a receiver peer select many sender peers from the available set of the candidate peers available in the overlay plane as presented in Fig. 2. Receiver peer intends to receive the requested video contents with improved QoS that can be achieved by improving the received video throughput and lowering the packets drop ratio [5]. Thus, best peer selection plays an important role in improving the overall QoS.

We focus to use Scalable Video Coding (SVC) [6] as an important component of the proposed network-aware IPTV services delivery. The characteristics of SVC make it more suitable for the heterogeneous environments in which the video stream is decodable dynamically in the spatial, temporal, and SNR resolutions. SVC truncates the original video into many different sub-layers, i.e. base layer and enhancement layers. The base layer provides the basic quality (QCIF with7.5 frame/sec.) of the video and is decode-able independent to layers. In contrast, the enhancement SVC layers are used to refine the video quality but these enhancement (enh.) layers are decode-able with reference to base layer and the other corresponding enhancement layers.

The cooperative mechanism based on multi criteria not only ensures the improved QoS but also improve the network utilization by guiding the receiver peer to select appropriate sender peers.

\section{PERFORMANCE EVAluATIONS}

We evaluated the proposed IPTV streaming over the collaborative framework using the network simulations using NS-2 simulator. This section describes the simulation results to determine the viability of the proposed mechanism.

\section{A. Simulation Model}

We consider an overlay network of participating peers that is constructed in accordance to the ISP's guidelines. ISP provides the information about the set of sender peers, aggregation nodes, and available network capacity of links. We distribute the SVC video layers among different sender peers. A receiver peer intends to receive the video packets from multiple sender peers for IPTV service, where the selection of the sender peers is influenced by the multiattribute mechanism implemented over the overlay plane.

We perform simulations to receive the overall SVC video quality CIF/CGS0 with $15 \mathrm{fps}$ at receiver peer. The receiver peer selects an individual sender peer to receive different SVC video layers. We truncated MPEG-4 trace file "Formula 1" into four SVC video quality layers where base layer offers $40 \%$ throughput (quality) of the original video, enhancement layer 1,2 , and 3 offers $25 \%, 20 \%$ and $15 \%$ throughput of the original video file respectively. It is obvious that no sender peer is contributing $100 \%$ quality of the video content, however if receiver peer receives all the four SVC video layers completely, the original video quality can be achieved. The simulation time was 60 seconds and the presented results are the average results of multiple runs of these simulations. We attached three "CBR sources" to overload the network that 
are started at time 5 seconds and stopped at time 55 seconds during the simulations and they inject constant throughput of 512. We performed these simulations for two scenarios.

- Network-aware Streaming Mechanism: In this scenario, participating peers are organized through explicit interaction between ISPs and P2P applications in the overlay plane. The peers inside the overlay plane are ranked on the basis of relative multi-criteria namely "RTT" \& "\# of hops" that is an indicative of the offered video quality and can be used as potential parameters in determining the geographical distances. Tracker provides this information to construct ISP-driven overlay network. Eventually, receiver peer use this overlay for the selection of multiple sender peers to receive SVC layers for IPTV.

- Random PULL-based Streaming Mechanism: In this scenario, the overlay network is constructed by the receiver-peer itself without considering any underlying network conditions. The receiver peer selects multiple sender peers from overlay network in order to "PULL" the requested video content for IPTV video service delivery.

\section{B. Simulations Results}

Fig. 3 presents the overall received video throughput from all the sender peers in both scenarios. We observe that the video packets received in our proposed network-aware streaming mechanism are $100 \%$ because the packet delivery is assured by the ISPs through the QoS enabled pipes. Furthermore, the best peer selection in network-aware streaming is also driven by the ISPs. The overall packet drop ratio for the total video quality is presented in Fig. 4.

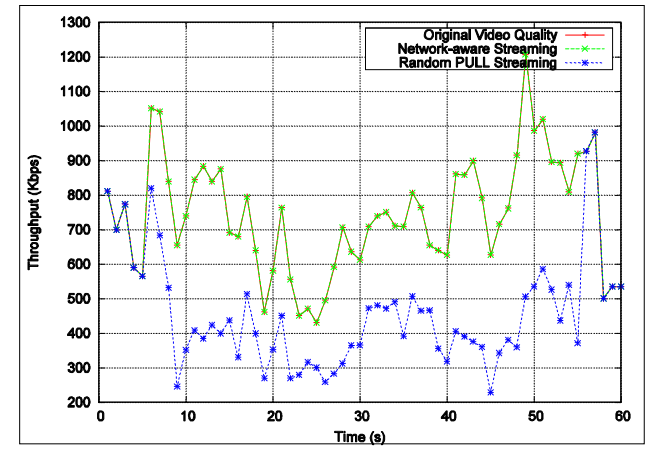

Fig. 3. Received Throughput.

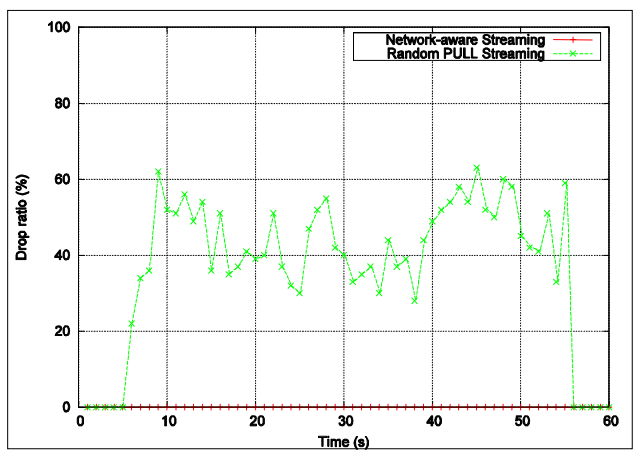

Fig. 4. Packets Drop Ratio.
The random pull based streaming does not guarantee any QoS for the received video for IPTV services because it chooses self agnostic random "e2e" content delivery paths. The heavy packet drop ratio in the said scenario is not acceptable for any real-time application.

We know that SVC layers have certain dependencies with each others for the actual decoding of the video content before their actual playback. In this situation, the video packets received in the receiver-side buffers for individual video layers cannot contribute to construct the original video quality if receiver peer does not receive video packets of lower SVC layer corresponding to the upper SVC layer. Moreover, network oblivious peer selection causes inefficient utilization of bandwidth, i.e. the allocated bandwidth is not fully utilized. Thus, we calculated the bandwidth utilization for all individual video layers as described in Eq. 1. Fig. 5(a), Fig. 5(b), Fig. 5(c), and Fig. 5(d) represents the viability of the individual SVC base layer, enh. layer 1, enh. layer 2, and enh. layer 3 respectively. The proposed mechanism show noticeable improvement in minimizing the e2e delay for content delivery and for application level QoS estimates. However, the results are not present due to space limitation.

The presented results show that video content delivery for IPTV over ISP-driven overlays not only improves the overall QoS but it also improves the utilization of available network bandwidth. In network-aware IPTV the video packets received during the streaming session contribute $100 \%$ utilization of allocated bandwidth whereas, the received video packets in random pull based streaming does not contribute to enhance the QoS but leads to inefficient utilization of bandwidth that utilizes around $60 \%$ of the allocated bandwidth. Thus, the results presented clearly highlight the viability of proposed ISPs \& P2P applications collaborative framework for IPTV services delivery.

\section{RELATED WORKS}

There have been a number of research and standardization activities going on for the collaborative frameworks for ISPs \& P2P applications. The P4P working group [7] has initiated to propose a protocol to establish better cooperation among ISPs and P2P applications. This protocol is an extension to the classical P2P framework. In this protocol, two types of trackers called "iTracker" and "pTracker" have significant role. The "iTracker" have close association with the ISPs and provides the information for the underlying network topology, whereas, "pTracker" keeps track of the participating peers and this information is provided to the newly joining peer. P4P employs the static information for these trackers, however our presented solution utilizes more dynamic information. The detailed different of our proposed ISP-driven P2P framework with P2P and P4P framework is presented in section III.

A new IETF working group, Application-Layer Traffic Optimization (ALTO) [8] has initiated activities for the standardization for P2P applications and ISPs interactions. 


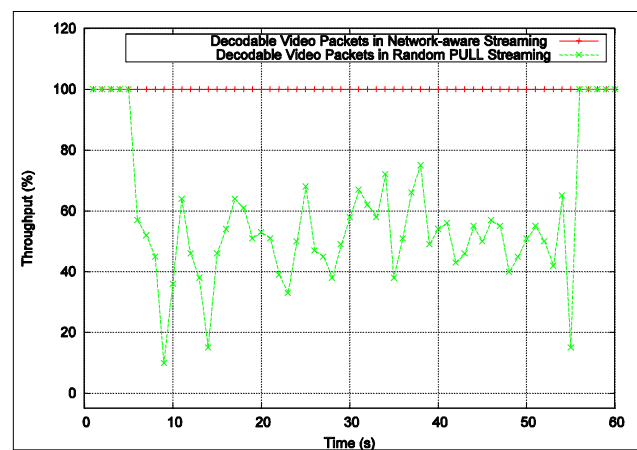

(a) Base Layer

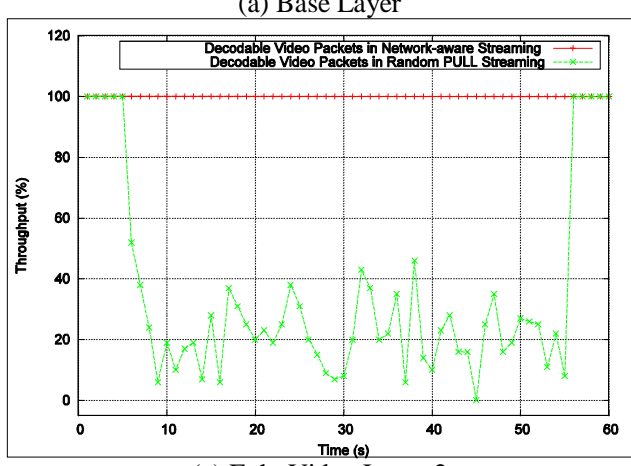

(c) Enh. Video Layer 2

Fig. 5. Viability of Bandwidth Utiliza

Initially, the ALTO group initiates discussions for overlay organization on the basis of the ranking of the peers to provide solutions for the network optimizations. However, the activities of the group are limited to network-aware service delivery.

In [9], a distributed ISP-friendly rate allocation algorithm for VoD applications is proposed instead of tacking the problem of topology building. The proposed algorithm intends to minimize the server load, unfriendly ISP traffic, and maximizing the peers perfecting. The solution utilizes the "iTracker" approach similar to the P4P framework and is applied over a mesh topology.

In contrast to the above studies, we present a framework for better interaction among $\mathrm{P} 2 \mathrm{P}$ applications and ISPs that is followed by the participating peers' organization in ISPsdriven overlay networks. The framework utilizes the end-toend knowledge of underlying physical networks on the basis of physical characteristics that enhances the overall system's performance. This ISP-driven overlay organization and peer selection can ensure the interests of both P2P application providers and ISPs. Moreover, it can be utilize to penalize the unauthorized peers passing through the ISPs domains.

\section{CONCLUSION \& FUtURE PERSPECTIVES}

In this paper, we have presented a framework enabling cooperation between ISPs and P2P applications for the network-aware IPTV video services delivery. This mechanism extends the functionalities of the available P4P and ongoing ALTO working group solutions to ensure the network-aware real-time services delivery over $\mathrm{P} 2 \mathrm{P}$ networks. The mechanism is evaluated using the network simulations compared to the random pull based streaming mechanism for IPTV service delivery. The proposed ISPs \& P2P applications collaborative

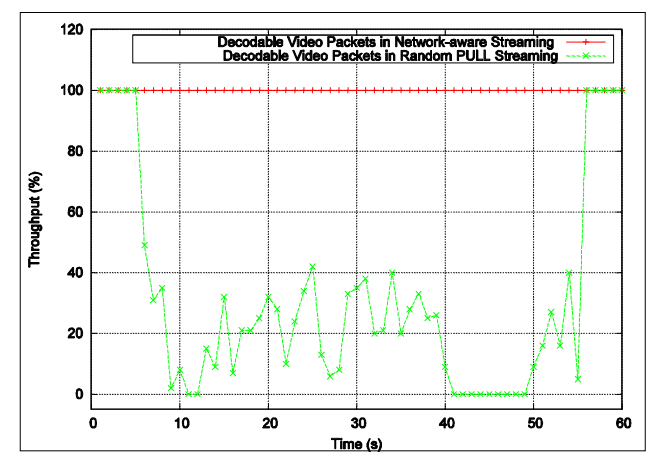

(b) Enh. Video Layer 1

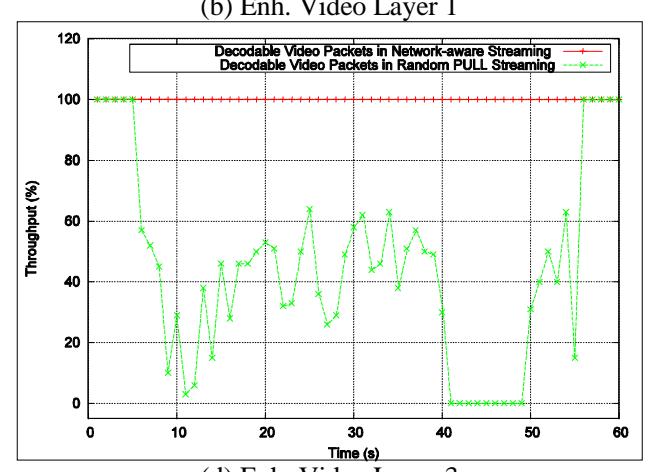

(d) Enh. Video Layer 3

lization for Received SVC Layers.

framework ensures QoS with P2P services perspective and efficient network utilization in ISPs perspectives.

In future, we intend to extend this framework for the network-aware streaming services delivery over large scale networks where the peers are located in different ISPs domains. We also plan to actually implement or emulate the proposed mechanism to conduct the real tests while considering multiple attributes for the best peer selection.

\section{REFERENCES}

[1] P. Pietzuch,, J. Ledlie, M. Mitzenmacher, and M. Seltzer, "NetworkAware Overlays with Network Coordinates". In Proc. of 1st Workshop on Dynamic Distributed Systems (IWDDS'06), 2006.

[2] X. Fu, H. Schulzrinne, A. Bader, D. Hogrefe, C. Kappler, G. Karagiannis, H. Tschofenig, and S. Van den Bosch, "NSIS: a new extensible IP signaling protocol suite". In IEEE Communication Magazine. Vol. 43. Issue. 10. pp. 133-141. 2005.

[3] S. Das, V. Narayanan, and L. Dondeti, "ALTO: A Multi Dimensional Peer Selection Problem”. Internet Draft. Network Working Group. Expires: April 26, 2009.

[4] C. L. Hwang and K. S.Yoon, "Multiple attribute decision making", In Proc of Springer Methods and Applications. New York 1981.

[5] M. Mushtaq and T. Ahmed, "Smooth Video Delivery for SVC based Media Streaming over P2P Networks". In Proc. of 6th IEEE Consumer Communications \& Networking Conference (IEEE CCNC 2008), Las Vegas, USA. 2008.

[6] J. Reichel, H. Schwarz and M. Wien, "Joint scalable video model JSVM8. Technical Report JVT-U202”, Joint Video Team, Hangzhou, China. 2006.

[7] H. Xie, A. Krishnamurthy, A. Silberschatz, Y. R. Yang, "P4P: Explicit Communication for Cooperative Control Between P2P and Network Providers", in P4PWG Whitepaper. Accessible online from http://www.dcia.info/documents/P4P_Overview.pdf 2007.

[8] E. Marocco, and V. Gurbani, "Application-Layer Traffic Optimization (ALTO) Problem Statement”. Internet-Draft, draft-marocco-altoproblem-statement-02, 2008.

[9] J. Wang, C. Huang, and J. Li, "On ISP-Friendly Allocation for PeerAssisted VoD”, in Proc. of ACM Multimedia 2008. 